Texas A\&M University- San Antonio

Digital Commons@ Texas A\&M University-San Antonio

History Faculty Publications

College of Arts and Sciences

1992

\title{
The Role of Adaptation in Archaeological Explanation
}

Michael J. O'Brien

Texas AઐM University-San Antonio, Mike.Obrien@tamusa.edu

T. D. Holland

Follow this and additional works at: https://digitalcommons.tamusa.edu/hist_faculty

Part of the Anthropology Commons

\section{Repository Citation}

O'Brien, Michael J. and Holland, T. D., "The Role of Adaptation in Archaeological Explanation" (1992). History Faculty Publications.

24.

https://digitalcommons.tamusa.edu/hist_faculty/24

This Article is brought to you for free and open access by the College of Arts and Sciences at Digital Commons @ Texas A\&M University- San Antonio. It has been accepted for inclusion in History Faculty Publications by an authorized administrator of Digital Commons @ Texas A\&M University- San Antonio. For more information, please contact deirdre.mcdonald@tamusa.edu. 


\title{
THE ROLE OF ADAPTATION IN ARCHAEOLOGICAL EXPLANATION
}

\author{
Michael J. O'Brien and Thomas D. Holland
}

\begin{abstract}
Adaptation, a venerable icon in archaeology, often is afforded the vacuous role of being an ex-post-facto argument used to "explain" the appearance and persistence of traits among prehistoric groups-a position that has seriously impeded development of a selectionist perspective in archaeology. Biological and philosophical definitions of adaptation-and by extension, definitions of adaptedness-vary considerably, but all are far removed from those usually employed in archaeology. The prevailing view in biology is that adaptations are features that were shaped by natural selection and that increase the adaptedness of an organism. Thus adaptations are separated from other features that may contribute to adaptedness but are products of other evolutionary processes. Analysis of adaptation comprises two stages: showing that a feature was under selection and how the feature functioned relative to the potential adaptedness of its bearers. The archaeological record contains a wealth of information pertinent to examining the adaptedness of prehistoric groups, but attempts to use it will prove successful only if a clear understanding exists of what adaptation is and is not.
\end{abstract}

La adaptación, un venerable ícono en arqueología, desempeña a menudo el papel huero de argumento ex post facto utilizado para "explicar" la aparición y persistencia de rasgos en grupos prehistóricos-una posición que ha obstaculizado seriamente el desarrollo de una perspectiva seleccionista en arqueología. Las definiciones biológicas y filosóficas de adaptación-y por extensión, las definiciones de lo adaptado-varían considerablemente, pero todas ellas difieren por completo de las habitualmente empleadas en arqueología. La concepción predominante en biología sostiene que las adaptaciones son características que fueron moldeadas por selección natural y que incrementan la adaptación de un organismo. De este modo las adaptaciones se distinguen de otras características que pueden contribuir a la adaptación pero son el resultado de diferentes procesos evolutivos. El análisis de la adaptación procede en dos etapas: la primera, demostrar que una característica se encontraba bajo selección; la segunda, demostrar cómo funcionaba la característica en relación con los estados adaptativos potenciales de sus portadores. El registro arqueológico contiene abundante información pertinente para examinar la adaptación de grupos prehistóricos, pero los intentos de utilizarla solo tendrán éxito si se comprende claramente qué es y qué no es adaptación.

In "The Archaeological Study of Adaptation: Theoretical and Methodological Issues," Kirch (1980:101) points out that of all the social sciences, archaeology, because of the kinds of data bases it can generate, should be in a position to develop a methodological basis for the examination of cultural adaptation. He highlights the adaptational rhetoric that has enjoyed widespread use by archaeologists, including such terms as "adaptive systems," "adaptive trajectories," and "preadaptation," noting that "Given such frequent appeal to an adaptational framework, one might expect that anthropologists and archaeologists would have expended considerable effort on defining fundamental principles of the adaptation process. Such is not the case" (Kirch 1980:101).

Our position is that anthropology in general, and archaeology in particular, are not even close to having a developed set of methods for the study of human adaptation. We also believe that not only are the methods lacking but that a critical lack of understanding exists on the part of anthropologists and archaeologists of what adaptation and its closely allied concept of adaptedness (fitness) are and are not. This misunderstanding not only cripples attempts to identify and understand specific human adaptations but also calls into question our understanding of principles that have received

Michael J. O'Brien and Thomas D. Holland, Department of Anthropology, University of Missouri-Columbia, Columbia, MO 65211

American Antiquity, 57(1), 1992, pp. 36-59.

Copyright (C) 1992 by the Society for American Archaeology 
extraordinary treatment by evolutionary biologists and philosophers of biology, who themselves diverge sharply on the concepts of adaptation and adaptedness.

Most anthropologists would readily agree that the lack of congruence between biological and anthropological views of adaptation is epistemologically sound, since humans are such extraordinary organisms that as subjects they require a completely different philosophical and methodological approach. We argue, however, that human adaptedness and adaptation do not make us immune from natural selection and that despite historically based reasons for doing so, we cannot continue to equate the creative ability of humans with some bizarre, extraordinary means of escaping selective environments. Although adaptation is a consequence of selection acting on variation, it is only a limited consequence. Concerning humans, "It guarantees only that people who persist in less effective practices will not perpetuate their practices as well as will other people pursuing different practices. Adaptations, then, are sets of cultural practices that simply have allowed people to get by in the past, whose consequences have so far proved at least relatively less harmful to the perpetuation of their social groups than any others the people may have tried out" (Braun 1990:81, emphasis in original).

Lack of knowledge of the process of adaptation - and, by extension, of adaptedness-has led anthropologists to view the process as one by which groups of people add new and improved methods of coping with the environment to their cultural repertoire. Adaptation is, in one sense, a means of finding solutions to problems-of overcoming risk-but it in no sense is causal of future events, nor is it progressive. Where adaptation is invoked as an anthropological "explanation," it often is viewed as a product of vaguely referenced "selective agents"-elements of the natural or cultural environment - that force human groups to change or face decline or extinction. Unfortunately, the products of such exercises are vacuous, orthogenetic cause-and-effect statements that have the appearance and character of what Gould and Lewontin (1979) term "just so" stories.

Differences between how biologists and archaeologists view adaptation is an odd occurrence if (a) Alland (1975:63) is correct when he asserts, "In the early 1960s a group of anthropologists and archeologists took over adaptation from biology and ecology" and (b) Marks and Staski (1988:151) are correct in asserting that the "new archaeology" called for evolutionary thought. It may have made such a call, but apparently no one familiar with Darwinism answered. We argue that a Darwinian view of the archaeological record must be based on the premise that things contained in the record were parts of past human phenotypes (O'Brien and Holland 1990:35; see also Neff 1992). Importantly, the objects are not simply reflective of or related to phenotypes (Dunnell 1988: 23); they are parts of phenotypes in the same way beaver dams and bird nests are parts of phenotypes. As such, objects in the archaeological record potentially can contribute as much (and sometimes more) information regarding adaptedness and adaptation as can purely biological features. Because they are not units of reproduction, archaeological objects must be viewed in terms of replicative success (Leonard and Jones 1987), which can, through analysis, be tied back to the potential adaptedness of the humans responsible for the objects' replicative success or nonsuccess.

Importantly, this view does not place much emphasis on "culture." Although the concept has an important place in anthropological thought, without a clear understanding of what adaptation is and is not (and cannot be) little reason exists to discuss broader issues that rely on a solid understanding of adaptation. Whatever culture is, it includes more than "a set of behavior patterns that are learned by members of society from members of society and that are adaptations of their society to the environment" (Campbell 1966:288). We will show that (a) many cultural features, though they might contribute to adaptedness, are not adaptations and (b) other cultural features do not even contribute to adaptedness.

We emphasize repeatedly that the historical development of specific features, some of which will be adaptations, is critical to understanding the function of features. We strongly disagree with Alland's (1975:60) statement that "A processual theory of adaptation must account for continuity and change of evolutionary systems rather than the specific characteristics of the systems themselves. It must ... generate transformational rules which can be used to explain and predict changes in behavioral systems with specific characteristics under stated sets of conditions." The only laws that interest us are laws of contingency. In other words, history matters. It is much easier to sit back and invoke 
an evolutionary model of change, with its attendant vague invocations of "selective pressure" and "adaptive response," than it is to begin to understand the "specific characteristics of the systems themselves." But the results of the two endeavors are incomparable.

When armed with a more-fundamental understanding of the concepts of adaptation and adaptedness, archaeologists can make solid contributions to the application of evolutionary theory to the study of previous cultures. Recent additions to the archaeological literature (e.g., Braun 1990; Dunnell 1978a, 1980, 1985; Leonard and Jones 1987; Marks and Staski 1988; Neff 1992; O'Brien and Holland 1990; Rindos 1989) suggest that archaeology is poised to make such contributions. But several critical misunderstandings-especially the mixing of traits that were and were not products of selection-have kept archaeologists from using the archaeological record to its fullest extent. Conceptual mixing of kinds of traits has seriously impeded any hope of using the archaeological record to understand human adaptedness and adaptation.

\section{THE CONCEPTS OF ADAPTEDNESS AND ADAPTATION}

"Adaptedness" ("fitness") and "adaptation," though related concepts, have distinct meanings. Conflation of the two stems in large part from the fact that the terms "adaptation," "adaptedness," and "adapting" sound like different words for the same thing. Sober (1984:211) notes whimsically that "In a simpler world, the process that produces adaptation would be certain to increase the level of adaptedness. In a simpler world, an organism's 'adapting' to its environment by modifying its behavior and physiology would be the same kind of process as a population's 'adapting' to its environment by modifying its genetic composition." This, as we discuss below, most decidedly is not the case.

Definitions of adaptation and adaptedness in the evolutionary-biology literature are so numerous that they would easily fill the space taken up by this paper (see Krimbas [1984] for several). For reasons explained in more detail later, we define adaptedness as the state an organism is in, relative to its conspecifics, as a result of its evolutionary history. We follow Futuyma's (1979:308) definition of adaptation - "a process whereby the members of a population become suited over the generations to survive and reproduce" - and Ayala's (in Dobzhansky et al. 1977:498) definition of adaptations"features of organisms that have come about by natural selection because they serve certain functions and thus increase the reproductive success of their carriers." Because organisms relate to the environment on a constant-and constantly changing - basis, adaptedness must be viewed in an ahistorical sense; adaptation and adaptations must be viewed in a historical sense. Importantly, in the case of humans, because of their ability to transmit information intragenerationally, the historical development of adaptations may be short-lived. This is in clear contrast to other organisms.

Burian (1983:288) makes an excellent distinction between adaptedness and adaptation: "Fleetness contributes to the adaptedness of a deer (or makes the deer better adapted) if, and only if, other things being equal, it contributes to the solution of a problem posed to the deer-for example, escaping predation. Fleetness is an adaptation of the deer if, and only if, the deer's fleetness has been molded by an historical process in which relative fleetness of earlier deer helped shape the fleetness of current deer." This leads us to two critical points. First, adaptations were not necessarily evolved to solve a problem for which they later were well-suited to solve. In the above example, the mechanical equipment that led to fleetness was not evolved to make deer fleet, it simply led to fleeter deer. We return this point later. Second, adaptedness is not necessarily a product only of adaptation. Said a bit differently, because a trait increases the adaptedness of its possessor does not qualify it as an adaptation.

Surely such a distinction runs counter to the way in which the term "adaptation" is used in anthropology. How can we say that something that benefits an organism, i.e., something that contributes to its adaptedness, is not an adaptation? To be fair, some biologists and philosophers (e.g., Bock 1980; Ruse 1971) argue that anything that contributes to adaptedness is an adaptation. But not all traits have the requisite causal history to be adaptations (Brandon 1990:41). In other words, they were not shaped by natural selection. The interesting question becomes instead, Can 
we infer something about the evolutionary process by looking at those special effects of evolution that we term adaptations?

We should take heart in the fact that even Darwin wrestled extensively with adaptation, searching for a schema that took into account both (1) the perceived goodness of fit between organisms and their environments (the fact that organisms lived and reproduced in environments-and their offspring reproduced - was accepted as prima facie evidence that there was indeed a fit) and (2) the observation that organisms changed through time. Darwin based his early theory on the idea of limited perfect adaptedness (see Ospovat 1981; also Burian 1983), the basic tenet of which held that organisms are constrained by their structure and constitution, but within those limits organisms are perfectly adapted to their environment. That is, organisms are designed to meet and overcome specific environmentally induced hurdles. Selection was afforded the secondary role of urging populations to become perfect or face extinction.

By 1857 Darwin had developed the notion of relative adaptedness later used in On the Origin of Species (1859):

Once Darwin came to realize that variation is ubiquitous and largely undirected with respect to the needs of the organism, he was forced to employ a relative concept of adaptedness. .. All organisms face a multitude of problems bearing on survival and reproduction. If they all vary (at least slightly) in virtually all their features, then typical organisms are not perfectly adapted. Some, however, are at an advantage with respect to others - that is, they are better designed to meet the expected or expectable challenges of the environment [Burian 1983:292].

A key to understanding Darwin - and by extension adaptedness and adaptation-is keeping in mind that by 1859 the key to his theory of natural selection was differential (specifically intraspecific) reproductive success. In fact, Darwin's theory was designed to explain differential reproductive success, i.e., why different individuals leave different numbers of offspring in succeeding generations. In the process, it ultimately explains the origins and maintenance of adaptations in nature (Brandon 1990:9). Darwin explained differential reproductive success through reference to Thomas Malthus's (1798) notion of the "struggle for existence"1 (or, in the terms of Herbert Spencer, whose words Darwin later adopted, "the survival of the fittest").

Malthus may have influenced Darwin, but the type of struggle postulated by Darwin was distinctly different from that described by Malthus (Sober 1984:17). The Malthusian struggle involves individuals competing for the same scarce resources. The Darwinian struggle, though it may on occasion involve intraspecific and interspecific competition for resources, is more of a competition between an individual and the environment. Sober compares the difference between the two conditions to the difference between golf and tennis. Both games have winners, but in tennis a point scored by one player means a point not scored by the other player. Not so in golf, where each player's score is independent of those of all other players. 'In Darwin's 'large and metaphorical sense,' all that matters is that players end up with different scores - that they do unequally well in the 'struggle for existence" "'(Sober 1984:17).

\section{Adaptedness and the Problem of Tautology}

Anyone with even a passing knowledge of evolutionary biology is familiar with the controversy over the perceived tautology implied in Darwin's "survival of the fittest" (e.g., Bethell 1976) and efforts to clear up misconceptions contained therein (Brandon 1978, 1990; Burian 1983; Ghiselin 1969; Gould 1976; Mills and Beatty 1979; Sober 1984; Williams 1986). Two red herrings have been slipped into these arguments. The first is that tautologies are inherently bad. But theory is full of tautologies. In fact, tautology is a characteristic of theory, but rarely have scientists worried so much about that point as they have about fitness. The second red herring is found in continued focus on the inadequacy of the term "survival of the fittest." It should be clear to anyone that the term does not do its intended job. If we somehow could rank all organisms within a group from the most fit to the least fit, only one of them would emerge as the fittest in the true, superlative sense of the word. Thus only one organism would survive. As Darwin himself figured out, there was no such thing as a perfectly adapted organism, and fitness had to be examined in a relative 
sense, organism against organism. Mayr (1982:589) wryly points out that "Selection cannot produce perfection, for in the competition for reproductive success among members of a population, it is sufficient to be superior and not at all necessary to be perfect." In other words, the hallowed phrase becomes "survival of the superior," with superiority determined by which organisms (actually their blood lines) persist and which do not.

The important issue is how to compare the relative fitness of organisms, namely does fitness apply to the actual reproductive success of organisms or does it apply to the propensity (see Mills and Beatty 1979) for organisms to be reproductively successful? In other words, at what point is fitness measured-before or after (and maybe long after) an organism reproduces? Clearly Darwin equated reproductive success with fitness - the ability to produce viable offspring (in current terminology either [1] the genetic contribution made by organisms to succeeding generations, measured in terms of the number of offspring, or [2] the average number of offspring left by members of a group of similar organisms). By Darwin's early account, organisms either were fit or unfit in an absolute sense-the features they contained either got the organism through environmental peril or they did not. Even a careful reading of Darwin yields some ambiguity as to what he later meant by the term "fitness," i.e., after he had abandoned the notion of "limited perfect adaptation." Did he mean the actual (realized) contribution made by organisms to succeeding generations, or did he mean the potential contribution that organisms could make? Differences between these two positions may appear to be subtle, but they most decidedly are not.

Realized fitness simply refers to the fact that organism $a$ is more fit (better adapted) than organism $b$, if in fact organism $a$ outreproduced organism $b$ in a specific environment. The statement then becomes, If $a$ is better adapted than $b$ in environment $E$, then $a$ will outproduce $b$ in $E$. "Degree of adaptation, in this sense, is an empirical property of the organism (or of the class) in question, but one which can only be known post hoc" (Burian 1983:299, emphasis added). It is ironic that this concept has been labeled "Darwinian fitness," since as Burian (1983:299) points out, "Darwin almost certainly meant the phrase 'survival of the fittest' to stand for the tendency of organisms that are better engineered to be reproductively successful" (emphasis added). By adding the word "probably" to the above if-then statement, we overcome Burian's objection that what Darwin really meant was the tendency. Thus we have Brandon's (1990:11) view that the underlying tenet of Darwin's natural selection is simply, "If $a$ is better adapted than $b$ in environment $E$, then (probably) $a$ will have greater reproductive success than $b$ in $E$." This definition can be termed expected fitness.

We advocate focusing on potential (or "propensity for," to use Mills and Beatty's [1979] terminology) adaptedness instead of on realized adaptedness. This is exactly the position that Fisher (1985; see also Ghiselin 1966, 1969) takes when he argues that the "tautology problem" exists because, under some definitions of adaptedness, adaptedness and natural selection are viewed as dependent on one another instead of as related but separate constructs, each having its own theoretical and empirical basis.

\section{Selection and Drift}

Is adaptedness a product only of selection, or do other evolutionary processes, such as chance (drift), play a role? If drift does play a role, how significant is it? A related question is, Do selection and drift always produce better-adapted organisms? Selection, which we consider to be more of a process than a force, ${ }^{2}$ is, at base level, one kind of " "biasing effect' in the composition of a population" (Sober 1984:29), i.e., it is but one "cause" of evolution. Simply put, selection is a "weeding-out" process that leads to the differential reproduction of transmissible (by whatever means) traits in a succeeding population. Selection is completely dependent on variation as a fuel-there can be no selection without variation among a group of organisms. Important as variation is, it is in no sense causal of anything: "variations may be compared with building materials, but the presence of an unlimited supply of materials does not in itself give assurance that a building is going to be constructed" (Dobzhansky 1982:119).

An important but all-too-often-overlooked point is that selection operates on individuals and not on traits. Various features allow organisms to persist and reproduce in a certain environment, but 
it is the organisms and not the features that are under selective control. This is not to say that there cannot be selection for properties, but a critical distinction must be made between selection for properties and selection of objects (organisms). The important point is that "selection of" does not imply "selection for." "Selection for" is, as Sober (1984:100) notes, "the causal concept par excellence"; "selection of" pertains to the effects of selection: "To say that there is selection for a given property means that having that property causes success in survival and reproduction. But to say that a given sort of object was selected is merely to say that the result of the selection process was to increase the representation of that kind of object" (Sober 1984:100, emphasis in original).

In evolutionary biology several distinct kinds, or more properly modes, of selection are recognized-among them stabilizing, directional, disruptive, and frequency dependent-and each produces different results in succeeding generations. Our guess is that among humans the effects of each of these are, theoretically, observable within a single generation. The one on which we will focus most attention is directional selection, where there is selection for a single formerly peripheral phenotype or phenotypic feature. The net result is to increase that phenotype or trait relative to its alternatives and thus to move the distribution curve in a specific direction. Stabilizing selection, on the other hand, acts to reduce the number of new variants that arise, in essence selecting phenotypes or features that are intermediate to the extreme variants. Disruptive, or diversifying, selection produces a coexistence of two or more phenotypes within a population. Frequency-dependent selection, in which the fitness of a phenotype is dependent on its frequency within a population, produces, in contrast to the other modes of selection, a complex, inconsistent relation between phenotype and adaptedness. Frequency-dependent selection probably plays a role in all selection (Futuyma 1986:166), but this mode must be operating constantly among humans, especially given that the frequency of humans within a group significantly alters the nature of the selective environment in which the individuals function.

Unlike selection, drift in its many guises is the random component (not to be confused with stochastic processes, as we note later) in evolution. For example, an allele for a trait not under selection can drift randomly in frequency across generations of a population until it becomes fixed or reaches zero and disappears. Drift also can refer to chance events among organisms in a population that affect the frequencies of different phenotypes and therefore of individual phenotypic traits. In theory, drift is an easy concept to understand, but in practice its effects often are difficult to distinguish from those of other evolutionary processes. Understanding drift is basic to understanding adaptedness and adaptation, since, as we discuss in more detail later, drift cannot produce adaptations but it can affect adaptedness. Since adaptations lead to adaptedness, we run the risk of misidentifying features as adaptations if we misread the processes responsible for the features. Although "adaptedness is a probabilistic disposition, so that greater relative adaptedness regularly leads to greater actualized [realized] fitness. . . . chance can intervene and break that regular connection" (Brandon 1990:45).

\section{Adaptations and Nonadaptations}

Features are adaptations only if they evolved because there was selection for organisms that carried the features. But what of the converse? Should every trait that has become prevalent because of selection be counted as an adaptation? Lewontin (1978; see also Gould and Lewontin 1979) says no, adding the proviso that features that arise by selection also must cause an increase in adaptedness. Part of his argument is sound and part is not. He argues, correctly, that features that arise in a population do not necessarily contribute to the adaptedness of constituent members. But his insistence that to be an adaptation a feature must contribute to the adaptedness of its possessors is linked to the erroneous view that adaptations are features that will, for all times, increase the adaptedness of possessors instead of as features that once were beneficial to possessors. In other words, "A trait can be an adaptation for performing some task, though performing that task in the present environment confers no benefit. The point is that it was beneficial" (Sober 1984:199). In other words, a trait evolved as a response to selection against organisms without the trait.

Note that the key word here is "evolved," which embodies the concept of cumulative change over time. We are not saying, obviously, that a trait began because an organism or group of organisms 
needed the trait. We simply are saying that selection, by means of differential reproduction, in essence "created" the trait. Organisms that had the characteristic did better in a certain selective environment than did organisms without the characteristic.

Brandon (1990:41) perhaps says it best:

A trait may appear with one mutation. Such a trait is not an adaptation regardless of its effect on the adaptedness of its possessor. If the trait does increase the adaptedness of its possessors and thereby increases in frequency in the population it will then become an adaptation. But its initial appearance is explained by the chance mutation. Thus at that point it is not an adaptation. More interestingly, traits may actually evolve, that is, be the product of an evolutionary process, and still not be adaptations. The process is evolution by random drift.

Adaptive features, i.e., traits that increase an organism's adaptedness, take time to evolve (biologically) within a population, regardless of the mechanism(s) involved. Therein lies an interesting problem for biologists: How can organisms "predict" what will be "needed" in future environments, especially in light of the rapidity with which selective environments can change? The answer is, of course, they cannot predict anything. Given the extremely slow rate at which advantageous traits arise (and even then they may not be heritable), and the extreme unlikelihood that they will reach fixation (a rate of occurrence that is twice the selection coefficient, itself a small number), it seems miraculous that any trait ever reaches the point where it becomes characteristic. It seems that the same or similar mutation would have to be introduced repeatedly and that the environment would have to be stable enough for the trait to go to fixation.

Lewontin (1978) uses the term "tracking" to denote the relation between a population evolving under natural selection and its environment. In reality, there is no perfect fit between the two. Since selective environments rarely are stable, information available to the population is always slightly out of date so that in actuality a population is tracking a past environment. The faster the selective environment changes, the more out of sync the population will be with the environment. Under rapid environmental change, selection can hardly act to improve adaptedness (Sober 1984:174); evolution by natural selection requires periods of environmental stasis, not constant upheaval. Van Valen (1973), coining the term "Red Queen hypothesis," posits that the environment changes just fast enough to prevent organisms from gaining on it, while adaptation keeps organisms from lagging too far behind. This reminds us of Braidwood's (1967:80) observation that Paleolithic cultures of Europe after the Pleistocene "changed just enough so that they would not have to change."

The point we are making is that features that evolved in one selective environment (or in a series of consecutive environments) may once have been adaptations. When the environment changed, they may no longer have been adaptations, i.e., they may no longer better adapt an organism and may in actuality be detrimental to its survival. In other words, "In order to qualify as an adaptation, a feature does not have to be currently undergoing active directional or stabilizing selection. If variation for a feature is not present, there can be no selection for that feature, and it does not thereby cease being an adaptation. It is only necessary that the feature be a component of a causal interaction of which the average effect, over all the contexts in which the feature occurs, is an enhancement of reproductive potential" (Fisher 1985:126, emphasis in original). Timing, in essence, is everything. The bottom line is that adaptations lead to increased adaptedness at specific times. This point is critical. Adaptation and adaptedness are complementary concepts, but "The former looks to the past, reflecting the kind of history that a trait has had. The latter looks to the future, indicating the chances that organisms have for survival and reproductive success. These retrospective and prospective concepts are mutually independent" (Sober 1984:210).

Gould and Vrba (1982) coined the term "exaptations" to refer to features that, after some period, come under selective control (or "become functional," to use parlance that we will employ later). "Aptation," also coined by Gould and Vrba (1982), refers to a trait that increases the adaptedness of possessors but that did not evolve because of that use. "Aptation" and "exaptation" together form what biologists for decades have called "preadaptations," a term that Gould and Vrba note suggests planning and foresight. But the distinction among "adaptation," "aptation," and "exaptation" is not always cut and dried. Brandon (1990:172; see also Fisher 1985:123-124) makes a 
point that is worth considering: Suppose a feature, because of some specific use, comes under selective control. Later in the lineage of the possessors, the trait, which no longer is under selective control, is co-opted for another use. It is then an exaptation. Suppose also that because of this new use the trait remains in the population for many generations, under the control of stabilizing selection. The trait has not changed, due in part to the role of stabilizing rather than directional selection. Since the trait is under selective control, Brandon views the trait as an adaptation.

Another class of features should be noted, though we are hard-pressed to devise a category for them. They usually are linked in the literature to discussions of pleiotropy, though they have more to do with architectural and engineering design. Gould and Lewontin (1979) point out two examples-the human chin and architectural spandrels-and show what the results would be if one decided that those indeed were adaptations. For example, the chin is simply the result of selection for other components of the jaw and not an adaptation. We recognize the chin as a facial landmark, but in actuality it hitchhiked its way to prominence through the force of selection working within certain architectural and engineering constraints on the mandibular portion of the phenotype. Similarly, architects did not build domed-roofed churches to create small triangular spaces (spandrels) in which to place paintings of the saints. Rather, spandrels are engineering by-products (that subsequently were filled with paintings) of mounting a dome on rounded arches. If there is another way to mount a dome on rounded arches without creating small, tapered spaces, we are unaware of it. The caveat here is that engineering studies-both of features and the organisms containing the features-are needed before we can recognize the features as more than simply tag-alongs.

This is precisely why Sober (1984:197) makes the following observation: "It follows that the concept of adaptation needs to be understood in terms of the idea of selection for properties; the idea of selection of objects will not suffice.... If a neutral trait is pleiotropically linked to an advantageous one, it may emerge because of a process of natural selection. It was selected, but this doesn't mean that it is an adaptation. The reason is that although it was selected, there was no selection for that trait" (emphasis in original). Sober is not suggesting that traits as opposed to organisms are the units of selection. He simply is attempting to highlight the causal emphasis that the phrase "selection for properties" connotes, i.e., that selection for and against properties produces evolution. "Selection of" has more to do with general adaptedness. Although we may be interested in differences in adaptedness among individuals, the presence of differences tells us nothing about the origin of those differences.

As a final consideration, we should point out that confusing the sequence of appearance of traits, i.e., what evolved from what (trait polarity), probably occurs as often in archaeology as in biology. If traits in question cannot be ordered chronologically, i.e., derived traits separated from primitive traits, we have little or no hope of ever knowing whether we have identified adaptations, let alone of ever knowing what the functions of the adaptations were.

\section{Determining Traits To Be Adaptations}

How do we determine that a trait is an adaptation? In other words, how do we know that a feature is the product of selection as opposed to the product of drift? Also, can we show that a certain trait, at a certain time, increased the adaptedness of the possessor(s)? These clearly are questions of cause and effect. If we accept that selection produces (causes) adaptations, which lead to increased adaptedness of the possessor(s) at a particular time, what can we infer about the selective process by examining adaptations? But doesn't this lead us back to the first question: How do we identify adaptations? In other words, without clear knowledge that a feature is an adaptation, how can we say anything about the process of selection, since drift may have created the trait? Is the whole exercise so difficult conceptually and methodologically that it is beyond our capabilities? Although we do not deny that the issues surrounding this problem merit considerable care and study, they do not pose insurmountable problems.

We might begin with the notion that adaptations were evolved for some purpose and that if we can (a) identify a function for a feature and (b) show how its possessors were more fit (relatively) than those organisms without it, then perhaps we can infer that we are looking at an adaptation- 
a feature that came under selective control. But there are biologists who have rained extensive criticism on those who view adaptations as solutions to problems and who set about to discover the functions of traits. Perhaps the most vocal are Gould and Lewontin (1979), who in their oftenquoted article "The Spandrels of San Marco and the Panglossian Paradigm: A Critique of the Adaptationist Programme" unmercifully attack studies that attempt to show the functions of organismic features (see also Lewontin 1986). They contend that organisms can be studied only as integrated wholes and that phylogenetic history, pathway development, and engineering constraint should be considered when attempting to identify adaptations and their functions. Their mostsignificant complaint is that many analyses result in adaptive stories that, while plausible, are untestable. Their view of the adaptationist program, however, is based on an erroneous belief that most studies of adaptation are reductionist in scope-traits are divided and subdivided into smaller and smaller increments and are continually searched to find a function for each unit.

But most adaptationist programs-including those in archaeology-do not operate this way (Mayr 1988:152-153). Properly conceived and carried out, a functional program is entirely appropriate. We hasten to point out, again, that function is at the heart of evolutionary change. Mayr (1988: 154 ) is right, of course: "the student of adaptation has to sail a perilous course between a pseudoexplanatory reductionist atomism and stultifying nonexplanatory holism." It would appear that to escape the criticism of Gould and Lewontin one not only must correctly identify adaptations as such but must then identify the proper function of the feature relative to the organism as a whole. If this were the case, however, few adaptations would have been identified and even fewer identified correctly. Contrary to their view, nothing is inherently wrong with the "try and try again" approach to analyzing features, whether they are adaptations or nonadaptations. What is needed is a method of falsification so that successive propositions can be constructed and their implications tested.

One method of analyzing features is through the examination of engineering design. It is clear from even a casual reading of On the Origin of Species that Darwin was fascinated with engineering design - both in terms of the features created and the process that created the design - to the point of referring to some features as "organs of extreme perfection and complication." Though he was ignorant of the mechanics of inheritance, Darwin saw no reason to treat even the most complex of features as anything more than cumulative modifications of earlier features-features that conferred some potential reproductive advantage on their bearers. These features, or adaptations, are the links between an organism and the environment in which the organism lives. To Darwin, the better engineered a feature was, the more potentially reproductively successful the organisms bearing that feature would be.

Any engineering-design study must keep several points in focus. First, as we have seen, not all features are adaptations, i.e., are the products of selection. Second, features that once were adaptations may not have been adaptations under different environmental regimes. Third, simply because a feature evolved as an adaptation does not imply that the feature was an end-all solution to a problem. The evolved feature may have "solved" one problem for the organism but in doing so may have created other problems. Still, as Sober (1984:81) notes, "a careful engineering analysis can in principle permit us to make reasonable judgments about fitness differences in advance of finding out who lives and who dies" (emphasis added).

Sober (1984:81-82) offers another strategy for analyzing features-a process he labels "bootstrapping": "One may begin by investigating fitness ex post facto-that is, by looking at differences in survival and reproductive success in one population and reasoning that these were a consequence of certain fitness differences. Then, when confronted with a second, similar population, one might be able to use one's previous experience to reason in the opposite direction. The hypothesis would be that the characteristics that determined fitness differences in the first population also do so in the second."

Both methods of determining whether features are (were) adaptations rest on inference. Engineering studies, for example, can show us how certain structures confer the ability to run fast, but we make the inference that the ability to run fast gave organisms a selective advantage over those that did not. Similarly, we can relate the presence of water-retention devices in vascular plants to their distribution in xeric environments. We base these inferences on our knowledge of how the world 
operates. Sometimes we might be wrong in our inference. Bootstrapping can be used to examine features in one group vis-à-vis survival and reproductive success, where our inferences are on solid footing, and then apply those results, inferentially, to another group. This appears to us to be the proper use of ethnographic data in attempting to solve select archaeological problems (O'Brien and Holland 1990:48).

\section{ADAPTEDNESS, ADAPTATION, AND THE ARCHAEOLOGICAL RECORD}

This brings us to the second theme of this paper. How do we use the ideas of potential adaptedness and adaptation, as defined here, to examine archaeological problems? At a more-general level, do these concepts apply to the study of humans? Our answer is emphatically yes, though we expect that not all anthropologists will agree. Few people familiar with Darwinian evolutionary theory have trouble accepting the premise that, except in a few technical ways (usually because of design constraints), selection is not directed toward some end. That is, selection is not what commonly is called "Lamarckian." To be precise, most people have no trouble with undirected selection when it applies to fruit flies, but when we start talking about humans, all bets are off. For when we speak of humans we are speaking of animals with intellect and will. Intention is, "if not the soul of Western man, a close approximation thereof" (O'Brien and Holland 1990:43). Humans are born actors and thinkers. As such, don't they have at their disposal the means to escape selection? On the other hand, under a "purely" biological perspective don't we completely discount the roles played by rationality and intent? The answer to both questions is, unequivocally, no. Steward (1956:72) sagely noted that "a specific invention is not explained by saying that man is creative." Instead of discounting human intention, selectionist theory assigns it the important role of introducing variation into a cultural system. Variation, not intent, is the significant component. In nonhuman biological evolution variation results largely from mutation and from the reshuffling of existing alleles during reproduction. In human evolution variation results in part from perception and intention. If it were the case that all outcomes of thought processes were those intended, then we have an excellent case of directed variation. But this is decidedly not so (see Reynolds 1984), since the simplest of "intended" actions can spawn an infinite number of outcomes and a dizzying array of variation.

At an intuitive level, no one would argue that a person who sits down to manufacture a stone tool does not have an intended outcome in mind. So in one sense-a trivial sense-we can speak of intent as a proximate "cause" of something. But of what analytical interest is such a statement? When we relate an evolved feature to the notion of intent, we close the book on further analysis. We argue elsewhere (O'Brien and Holland 1990:45) that some confusion surrounding the nature of selection relative to humans undoubtedly can be attributed to use of the term cultural selection(ism) (e.g., Rindos 1984, 1985, 1986), the basic tenet of which is that evolutionary processes can act independently on genes and on culturally transmitted behavior (the term "cultural selection[ism]" probably should be dropped from usage, despite its wide adoption [see Braun 1990:79]). Although we should never doubt Mayr's (1973:388) assertion that "Behavior is perhaps the strongest selection pressure operating in the animal kingdom" or Rindos's (1986:316) statement that learning is "one of the most important determinants of behavior," we should not assume that humans are governed by a different process than that which governs other organisms. If variation, whether by accident or design, confers increased adaptedness on an organism, and if the trait is inheritable or transmissible by any means, then all else being equal the trait will be represented differentially in the succeeding generation. What is important to the selectionist is that phenotypic (defined broadly) variation is available.

If we accept this position, then the question we asked earlier-How do we use the concepts of potential fitness and adaptation to solve archaeological problems? - should be inverted. The question now becomes, How can the archaeological record be used to analyze the fitness and adaptations of past groups of people? Further, what methods can we use to elicit such information? It seems reasonable to assume, given our premise that things in the archaeological record were parts of past human phenotypes, that the record contains fossilized traces both of features that bore directly on the potential adaptedness of their possessors as well as of features that did not. Additionally, some 
of those features were adaptations, and some were not. Step one in our analysis should be focused on separating features that affected adaptedness from those that did not. Step two should be focused on separating adaptations from nonadaptations.

\section{Adaptations and Nonadaptations}

Leonard and Jones (1987) make an interesting point about using traits to examine fitness. They distinguish between the success of the features (traits) themselves and the success of the trait possessors. Since features are not the units of selection (their possessors are), they must be viewed in terms of replicative, not reproductive, success. Leonard and Jones (1987:213-214) correctly note that "While the differential reproductive success of individuals may influence the expression of cultural variation, it does not determine it." Thus replicative success does not necessarily signal that a selective advantage has been passed to the possessors of the feature, nor does it imply that the feature was under selection.

Picking up on Leonard and Jones's notion of reproductive success, we note elsewhere (O'Brien and Holland 1990:50): "In terms of reproductive success, possession of a trait may make individuals more fit by giving them a selective advantage over individuals not possessing the trait. But if possession of that trait has no effect on the reproductive success of the possessor, then the trait is neutral" (emphasis in original). However, given our definition of adaptation and our emphasis on potential adaptedness rather than on realized adaptedness, we clearly no longer can subscribe to the point made in the last sentence. We would amend the sentence to read, "But if possession of that trait has no effect on the adaptedness of the possessor, then that trait is neutral; if the trait did not evolve because of selection, then it is not an adaptation."

Selective neutrality implies that an organism gains no selective advantage by possessing one state of a trait over another state of that trait, i.e., each state carries equivalent fitness values. Importantly, and we cannot emphasize this enough, neutrality of alternate states of a trait does not imply, as we discuss below, that possessing the trait carries no selective value. Also, neutrality is tied to a particular time, since, as we have seen, neutral traits can become nonneutral. Recall that not all traits that increase adaptedness are under selective control. Up to now we have ignored this subtle but important point. But this point, with the topic of neutrality, is so critical to archaeology that misinterpretation of them has led to massive confusion by archaeologists interested in developing and applying an evolutionary approach (e.g., Rindos 1989). The problem, in part, stems from misinterpretation of Dunnell's (1978a) dichotomization of function and style and his curious and perhaps counterintuitive linkage between style and neutrality. We elsewhere (O'Brien and Holland 1990) attempted to clear up the problem but were only partially successful.

The problem also stems from an implied equation, in the few instances where function and neutrality have been discussed in the archaeological literature (e.g., Dunnell 1978a, 1978b, 1980; Leonard and Jones 1987; Neff 1992; O'Brien and Holland 1990), of adaptations and "functional" traits and an equation of nonadaptations and neutral traits. Dunnell (1978a), for example, distinguishes between function and style both in terms of Darwinian fitness and of selective value. He uses function to refer to those forms that directly affect the Darwinian fitness of populations in which they occur and style to refer to those forms that have no detectable selective values. In other words, "style" refers to neutral traits. But what we have here is a shift in emphasis of criteria necessary for inclusion in the two categories. An increase in Darwinian fitness is used as the criterion for inclusion in the function category, while the absence of selective value is used as the criterion for inclusion in the neutral (style) category. But what do we do with features that increase the adaptedness of the possessor(s) but that are not products of selection? To bring the problem into focus and to avoid problems associated with the dichotomous terms "style" and "function," we create three categories of traits: (1) traits that are under selective control and that increase adaptedness; (2) traits that are not under selective control and that increase adaptedness; and (3) traits that are not under selective control and that do not increase adaptedness.

Clearly traits in category 1 are the products of selection. They are, by definition, adaptations. Traits in categories 2 and 3 are products of other processes, which we discuss below. Under Dunnell's 
use of the term "function"-as applied to traits that increase Darwinian fitness - traits in both categories 1 and 2 qualify, though it is clear from the contexts in which Dunnell uses the term that he is referring to traits in category 1, i.e., those that both contribute to adaptedness and are products of selection. Dunnell's use of "style" refers to traits in category 3-"traits" (in reality, states of traits) that do not contribute to adaptedness and therefore are neutral.

But can't "style" contribute to adaptedness, whether or not it comes under selective control? In one sense it can, and it is for this reason that confusion has reigned supreme over Dunnell's use of the term to imply neutrality. Style, as Dunnell (1978a) uses the term, is neutral only to the extent that at a given time any particular stylistic feature is as "fit" as any other stylistic feature. The critical point here is use of the word "feature" and the confusion it creates. It is precisely for this reason that we use the term "feature state" (or "trait state") instead of "feature" (or "trait"). What is important is that there may be several or many alternate states (attribute states of a specific dimension [see Dunnell 1971]) in which a trait can reside, with each state conferring equivalent (or, sometimes, nonequivalent) adaptedness to the possessor. Thus it is imperative to separate the concept of "style"-an ill-defined complex of traits and trait states-from the phenomena of "stylistic elements."

An example is the incision of circles, chevrons, or birds into moist exteriors of unfired pots. Two questions are relevant here. First, is it important that pots are decorated at all (the presence of decoration being a feature [trait])? Second, is it important that chevrons are used instead of birds or circles (the individual designs being states of traits)? It would make little sense to call a chevron an adaptation, but it might make sense to call pot decoration an adaptation within a given setting. We could construct several scenarios where loosely knit social groups spread across a landscape use decorative displays for social purposes-either for integration or information exchange (e.g., Braun and Plog 1982; Wobst 1977). By participating in the social-identification system of which the marked pots are a feature, a person might increase his/her adaptedness: Food can be shared in time of need, new mates are available, and so on. By not participating in the system, individuals (or a group of individuals) could be affecting their adaptedness relative to other individuals or groups in the region. But examining the role of pot decoration in this context is decidedly different from examining pot decoration abstractly. The mere fact that someone out of the blue decides to decorate a pot is, by itself, not a functional trait.

This raises a related point about selection and adaptedness. We might suggest that despite the wide range of decorative variants possible in the world, there are some that the groups using the pots find unacceptable. Or, more probable, there are variants that make no sense to the users. Thus there is an acceptable range of decorative variants available. As long as makers or users remain within the range, which we might expect could and would change over time (for example, by ca. A.D. 300 midwestern cooking jars no longer carried elaborate decorations), their adaptedness, at least relative to this one dimension, is not affected adversely. But pot makers or users who consistently defy the limits of acceptability could have their adaptedness affected. Again, what is important from the standpoint of potential adaptedness is not that the pots specifically have birds or chevrons on their exteriors-or that the pots are decorated at all-but that if the pots are decorated the makers and users know which elements are acceptable and act accordingly.

It isn't profound to say there are different scales at which features in the archaeological record can be examined and that a healthy dose of common sense is needed to reduce making errors of the first order. For example, without a perspective on the recurrence of cooking-vessel designs across broad regions of the Midwest, our picture of the life histories of ceramic "types" would be heavily biased. We could be left wondering whether there was some reason for a local group to decorate its pots for a while and then to abandon decoration. It is not too much off the point to note that lack of attention to detail at the regional level seriously impeded our understanding of post-ca. A.D. 300 developments in the midwestern United States. For years the received wisdom among archaeologists working in the Midwest was that the "Hopewell Interaction Sphere," characterized at many sites by nicely decorated vessels and the occurrence of exotic materials, came to a sudden halt as a result of groups becoming more isolated in their behavior and the concurrent lack of benefit from participating in the sphere. Braun $(1977,1985 a)$, however, shows conclusively that instead of becoming 
more isolated, at least in terms of ceramic similarities, post-A.D. 300 groups showed heightened homogeneity. The misconception was a result of analytical interest that for decades had focused solely on decoration instead of on manufacture and decoration.

Part of the confusion over style and neutrality undoubtedly stems from the fact that, as we mentioned before, the source of selection is tied to human intent. Anthropologists argue that humans select ceramic styles, dress lengths, and myriad other things based on culturally influenced choice. Thus, the argument runs, style cannot be selectively neutral. This problem is nothing more than the result of the same word having more than one meaning. Selection as an evolutionary process has little to do with cultural selection as often applied colloquially. What is meant in the latter sense is simply "choosing" one thing over another. Humans may be selective agents, but only if they affect the adaptedness either of themselves or of other organisms. For example, animal breeders are active selective agents. And, the seemingly capricious, but in reality patterned, choice by collectors of butterflies of one color or another is as potent an agent of selection as is the choice by any bird. In both cases the butterflies meet unhappy endings, and the gene pool of which they were a part is adjusted accordingly (O'Brien and Holland 1990:57). But this is very different from choosing one design element over another for vessel decoration. Clearly, the more-important analytical problem is understanding the pool of acceptable variation for given points in time and determining how remaining in the pool vs. straying outside it affects adaptedness.

To summarize, functional traits are seen as those that contribute directly to adaptedness and that are under selection. They are equivalent to traits in category 1 and are termed adaptations. Nonfunctional traits, such as design motifs on ceramic vessels, are not under selective control and do not affect adaptedness. They fall in our category 3 . But what about category- 2 traits, i.e., those that may affect fitness but are not under selective control? A biological example of such a trait would be a mutation. The corresponding nonbiological feature would be an invention, discovery, or similar product of a moment in time.

Not all such products of the moment affect adaptedness, especially those that arise and go unnoticed. Others very well could affect adaptedness, and many of them will go on to become adaptations. For example, a human who picks up an animal hide, punches a hole in it and puts it on may increase his adaptedness relative to others in his group. At that point, the hide is not an adaptation; it is merely a "mutation" relative to one human's phenotype. A number of sequences could follow. If, after a few generations, the person's offspring were living longer and producing more children than were their conspecifics, then the wearing of skins would become an adaptation. Or, if after a few weeks or months other members in the group noticed that the skin wearer appeared more comfortable than they, then they might start wearing skins. The wearing of skins might then be considered an adaptation. But suppose the skin wearer died the day after he started wearing skins, without telling anyone else how warm he felt? Then we have another example of a mutation that remained a novelty. ${ }^{3}$

Several interesting points come out of this example, perhaps the most important being we do not necessarily have to wait for generations to see if something is going to affect adaptedness or to come under selective control. The wearing of skins could become an adaptation almost over night. But, one might ask, don't we have to wait for succeeding generations to manifest themselves before we can decide whether the features are adaptations? Not if we are interested in potential adaptedness instead of in realized adaptedness. An engineering study could help us in determining whether an advantage was to be gained by wearing skins (of course, in this instance we can rely on common sense). One also could question whether our experimenting human really knew that by putting on a skin he was raising his body temperature to well above a life-threatening point and whether he could tell this to others in his group. This point really is irrelevant. If the trait spread because it was selected for, intentionally or not, and if the trait affected the adaptedness of the bearers, then it was an adaptation. No one in the group had to make any connection between body warmth and increased longevity.

In this single example we potentially have the makings of all three categories of traits. The wearing of skins, we can predict, affects adaptedness, regardless of the time we make the examination, i.e., when the feature is a mutation or when it is an adaptation. Thus the trait falls in category 2 and 
then, if it is acted upon by selection, category 1 . But does the kind of skin matter? Is bear skin superior to wolf skin, or does each confer an equivalent advantage to the wearer, i.e., are the relative fitnesses equivalent? (Certainly if we were debating the relative merits of bison hide vs. rabbit skin for use as shield coverings, then the answer would be clear.) Notice that the level of examination has shifted here from the trait itself-skin or no skin-to the attributes of the trait, similar to the shift seen in our example of pot decoration. Detailed engineering studies of different types of skins found in our imaginary archaeological record would have to be conducted before this question could be answered.

Concomitant with our engineering study, we might want to track changes through time in the occurrence of skins, both as a whole and of different kinds of skins. We propose that the manner in which frequencies of variants behave over time might be of more-than-passing analytical interest. Dunnell's (1978a:199) view of how such frequencies behave when they are either under or not under selective control is a starting point: "Traits that have discrete selective values over measurable amounts of time should be accountable by natural selection and a set of external conditions. Traits identified as adaptively neutral will display a very different kind of behavior because their frequencies in a population are not directly accountable in terms of selection and external contingencies. Their behavior should be more adequately accommodated by stochastic processes."

Variants under selective control $d o$ behave differently than do those that are not. Traits or alternative states of traits not under selective control can (a) drift through a population from generation to generation, their frequencies fluctuating randomly-sometimes in one direction, then another, and so on, or (b) come under selective control. Under the latter, mutations are either immediately acted upon by selection and the magnitude function shifts upward, or the feature or state drifts along, perhaps increasing in frequency, and then the magnitude function shifts upward. Under the former, given infinite time without selection, one of two outcomes is possible: Either the state will hit zero and thus be eliminated from the population, or it will become fixed. In reality, we find it difficult to believe that a neutral trait, or state of a trait, could ever reach fixation by chance without a drastic reduction of population numbers, as a result either of disaster or of fragmentation. By far the more likely outcome will be that the trait disappears from the population.

It is not, however, the random zig-zag curve that is of interest here but rather the familiar battleship-shaped curve that characterizes archaeological seriations. If most archaeological seriation curves are based on changes in stylistic frequencies, and if style is neutral and thus not under selective control, then why don't seriation curves exhibit the random zig-zag pattern shown by alleles not under selective control? We elsewhere (O'Brien and Holland 1990:54) claimed a close correlation between ordered seriation curves and the disorderly appearing, random-fluctuation curves. This was an overstatement on our part, we now realize, caused in large part by conflation of randomness on one hand and stochastic processes on the other. Stochastic processes are perfectly capable of producing orderly appearing events, whether they be design changes on pots or computer-generated clades of organisms (e.g., Gould et al. 1977). With clades, the result exemplifies the Markovian side of evolution's nature, where the form of each state depends in large part on the previous state. Archaeological "styles" also are Markovian in nature, i.e., styles are constructed from other styles, building on previous developments. On the other hand, the zig-zag pattern of allele fluctuations is nothing more than a graphic presentation of what in essence is the flip of a coin. Heads the allele frequency increases, tails it decreases. If the coin rests on its edge, the frequency remains unchanged.

As opposed to what happens to a trait not under selective control, a trait or, similarly, an alternative state of a trait that is being selected for, will begin at some arbitrary point above zero and increase in frequency at a steadily decelerating rate toward some optimal value. This gives selection its apparent "directional" component. Selection against the trait or state-the result of changes in the selective environment-reverses the trend and sends the curve downward. As selection favors new characteristics that arise, the fall-off in frequency of variants being replaced should be rapid. Given the rapidity with which variation can be generated among groups of humans, successive sigmoid curves with abrupt truncations should be the rule rather than the exception.

The decelerating curve applies equally to a trait or to a single state of a trait-depending on the level of analysis - under selection. States of traits (things) found in the archaeological record are not 
the equivalent of alleles, in the normal sense of the word, though at one level they can vary in the same way that alleles do. If, for example, we examine the kinds of temper added to cooking vessels through time in a region, we might find that over a 1,000-year span shell, sand, grog, and limestone were the major temper constituents, each with its own particular life history. What we need to track are the life histories of all temper kinds through time and to compare the curves. When we examine a fragment of tempered pottery, we will find one of two things: Either the vessel was tempered with a single material or with a combination of materials. Thus a vessel can be unimorphic or polymorphic with respect to temper. ${ }^{4}$

Pottery is an interesting class of materials to examine relative to adaptedness and adaptation, especially from the standpoint of nested units of adaptation (Kirch 1980:132). First, were prehistoric pots adaptations? Clearly it is not difficult to understand advantages that would accrue from having containers for transporting and cooking food. So if nothing else we can state that, from the standpoint of potential fitness, pots at least contribute to relative adaptedness in specific environments. Upon examining the life history of pottery and its meteoric rise in frequency once invented or introduced into various regions of the eastern United States, we would argue pottery was an adaptation. But even though we would argue pottery was an adaptation, we need to examine the life history of pottery in individual regions (O'Brien and Holland 1991). It is entirely possible for pottery, or pottery technology, to have been introduced into an area but used, for whatever reasons, only rarely. Thus it would not have been an adaptation for groups in that area.

Second, what technological factors relative to vessel construction might be adaptations? Braun's (1983, 1985b, 1987) work on Woodland-period cooking jars from west-central Illinois is a landmark study of the dynamics of prehistoric-vessel technology, especially of the covariation of technological dimensions. His early work (Braun 1983, 1985b) focused on tracking changes in vessel-wall thickness through time, a classic case of directional selection moving the mean slowly toward thinner walls. Braun argued that thin-walled vessels were being selected for their increased ability to conduct heat and their increased resistance to thermal shock. Further analysis (Braun 1987) showed that despite the neatness of this scenario, the logistic curve created by measuring vessel-wall thickness obscured many components that together created the curve, i.e., components that reflect the myriad decisions and limitations facing Woodland potters as they considered changing demands for performance characteristics in their products (Schiffer and Skibo 1987). An especially important technologythe production of extremely thin-walled bowls (almost always tempered with limestone)-was developed around A.D. 200-300, and though it lasted only a short time may have led to a transfer in technology to the production of cooking vessels with far-thinner walls per unit of vessel size than those manufactured previously. The general trends in vessel-wall thickness tracked by Braundespite the complexity of underlying logistical components-suggest thin-walled vessels not only contributed to adaptedness but also were adaptations (O'Brien and Holland 1991).

Third, was tempering-another technological dimension-an adaptation? Engineering studies (e.g., Feathers 1989; Grimshaw 1971) suggest the addition of temper greatly enhances a vessel's ability to withstand stresses that occur during firing. This fact, along with the almost-universal presence of aplastic inclusions in prehistoric vessels, suggests tempered vessels were adaptations (O’Brien and Holland 1991).

Fourth, were individual tempering agents adaptations? Based on our assessment of published and unpublished data on sherds and associated radiometric dates from one region of the Midwest, we suggest that in the lower Illinois River valley and in select portions of the Central Mississippi River valley vessels tempered with grit (various-size angular to subangular particles) were adaptations that later were replaced by vessels tempered with burned shell. Both appear to have had the requisite histories to qualify them as adaptations (Figure 1). In the case of grit tempering, angular particles were used from the beginning (ca. 500 B.C.) to temper first crudely made, thick-walled jars, and then thinner-walled vessels. Other materials sometimes were used, in most cases in conjunction with grit. By A.D. 600 in some areas shell was used as a minor tempering agent, but by A.D. 1000 it had become the predominant material used throughout the Mississippi River valley and over large portions of the Southeast. In other words, shell tempering began as a mutation that drifted along for several centuries before coming under active selective control. 


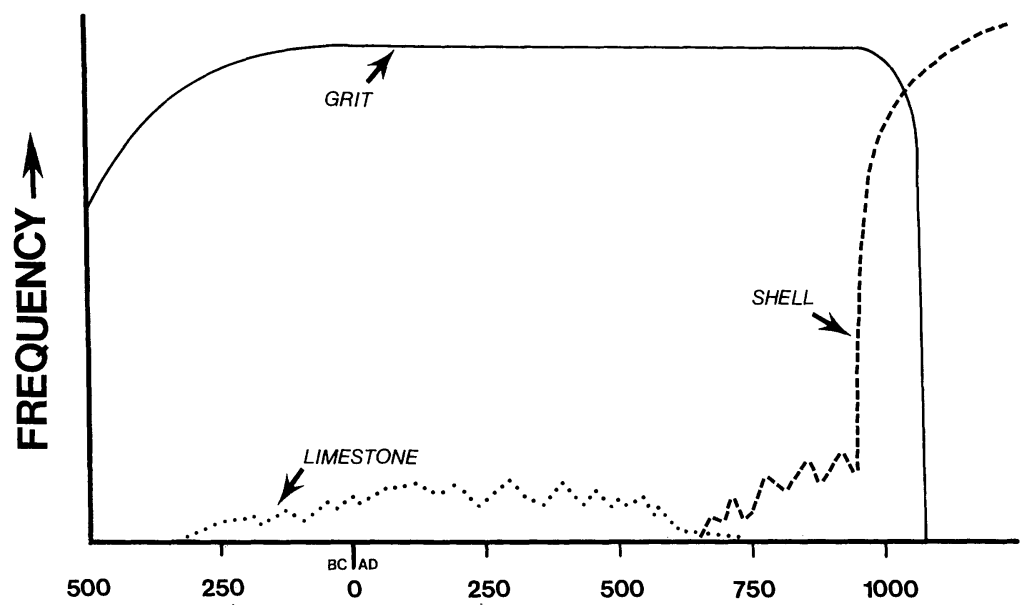

Figure 1. Hypothetical changes in frequency of grit-, shell-, and limestone-tempered vessels. Shell-tempered and grit-tempered vessels were under selective control; limestone-tempered vessels were not.

The case of pottery in the Midwest allows us to view two of the modes of selection mentioned earlier: stabilizing selection and directional selection. It also illustrates the manner in which each kind of selection operates. Although pottery was introduced into the southeastern United States during the third millennium B.C., with few exceptions (Reid 1984) it is recognized in the Midwest only in contexts that date after ca. 500-600 B.C. Pottery either was introduced into the Midwest or began as a mutation. Pottery manufacture in the Midwest accelerated in frequency around 500 B.C. In other words, there was strong directional selection for pottery manufacture. Concomitant with the rise in pottery production was the rise in grit as a tempering agent-again, a product of strong directional selection. After approximately 200 B.C., there was strong directional selection for thinner-walled cooking vessels, though the effects of this selection were ameliorated by another type of selection, stabilizing selection, which tended to eliminate certain variants that would have moved the thinning process along at a faster rate. Under stabilizing selection grit was kept as the predominate tempering agent, though other materials were in use in small amounts (Figure 1). Importantly, prehistoric potters knew about these other materials but rarely used them alone when manufacturing a cooking jar. Under directional selection, ca. A.D. 1000, shell as a temper began its rapid ascent.

We can pose some interesting questions regarding the directional selection and subsequent stabilizing selection of shell-tempered pottery, specifically questions regarding the kinds of advantages that shell tempering offered (Dunnell and Feathers 1991; Feathers 1988). Several studies (e.g., Million 1975; Stimmell et al. 1982) suggest that shell enhances the workability of sticky flood-plain clays, which were the matrix constituents of pre- and post-A.D. 900 ceramics in the Mississippi River valley. Late Woodland pottery was made from the same kinds of sticky clays-illites containing fine quartz, plagioclase feldspar, and traces of titanium dioxide (Feathers 1988)-but before ca. A.D. 900 sand and grog (in different areas) were the preferred tempers.

Feathers (1988) demonstrates that paste plasticity is increased through the addition of shell, apparently through increased flocculation caused by the presence of calcium cations. Increased plasticity eventually led to the production of a wide variety of ceramic forms. His tests also show an increase in the strength of shell-tempered ceramics over those tempered with sand. Also, the somewhat large platelike structures created by crushed shell are a better deterrent to crack propagation than are sand particles, thus causing fewer failed vessels during heating. The key to successful use of shell tempering is controlling firing temperatures below about $700^{\circ} \mathrm{C}$ - the point at which calcite, upon cooling and hydration, changes to $\mathrm{Ca}(\mathrm{OH})_{2}$ (Dunnell and Feathers 1991; Feathers 1988). Once prehistoric potters attained the skill needed to control firing temperature, shell-tempered pottery spread rapidly across the southeastern United States.

Has our discussion precluded the roles of human intent and choice played in the "evolution" of 
pottery? Not really. We would not argue that prehistoric potters did not set out to make certain forms, nor that they didn't experiment with tempers (see Rye [1976] for ethnographic examples of temper choice and desired results) and firing temperatures. We would argue, however, that it is impossible to know what went on in the minds of prehistoric potters and that regardless, the outcome is still measurable in terms of engineering design and by inference relative fitness. Occasionally one hears disparaging remarks directed toward archaeological studies that attempt to understand technological aspects of pottery manufacture-remarks stemming from a belief that little is to be gained from using high-tech equipment to measure things such as precise temperatures at which pots were fired, since prehistoric potters did not have similar equipment. But is retroactively measuring firing temperature of pots any different from measuring beak length in a group of finches or measuring the height of beaver dams? Such misplaced criticism grows out of a feeling that unless prehistoric peoples were aware of the variation inherent in their products, why would we want to measure it? Prehistoric potters obviously did not use pyrometers, at least as we know them, to measure firing temperatures, but they learned, eventually, to maintain temperatures below $700^{\circ} \mathrm{C}$. Again, the point is that selection is blind to the source of variation, regardless of origin. Pottery would be an adaptation whether it was introduced into an area or arose because of independent invention. Human intent neither adds to nor subtracts from increased adaptedness and adaptation. As Rindos (1984:4) notes, "Man may indeed select, but he cannot direct the variation from which he must select." Human selection-choice-because of the extremely imprecise way in which it replicates things, is a tremendous source of variation in its own right, producing new variants at a high rate. Any variant can affect adaptedness, and all are ripe for selection to begin operating on them.

\section{ADAPTATION IN A LARGER PERSPECTIVE}

Scientific approaches can be sound, but if no one uses them, either because of tradition or because they are too complicated, what use are they? We have argued here that the ways in which adaptation and adaptedness have been applied in archaeology and anthropology have little in common with the ways in which they have been applied in biology, and we have attempted to show how this noncongruity can be overcome. We do not think the approach is so complex that progress will not be made, though we suspect advances will be slow to come. Whether archaeologists break with tradition is irrelevant at this stage. There are, however, three points that could, and probably will, be leveled against the approach - points that need clarification before some researchers will feel comfortable in changing their views on adaptation.

First, is our focus on adaptations atomistic? Does a long and tedious examination of changes in temper inclusions in midwestern pottery-regardless of whether or not tempered pots are "adaptations"-really tell us anything about people? What about the bigger picture, which anthropology has always claimed as its objective? Our only answer to these questions is that in the end such detailed examinations will tell us considerably more about prehistoric peoples than will reference to vaguely defined terms such as "adaptive plateaus," "preadaptive strategies," and the like. It may sound trite to say that long journeys begin with but single steps, but that is exactly what faces us relative to the study of human adaptation. To understand the intricate process of adaptation and the ever-changing state of adaptedness, we need to establish as many small reference points as possible-reference points that eventually can be used to construct temporal curves for the examination of trajectories taken by various traits and their states.

We have, by necessity, limited discussion to analysis of individual traits and various ways of determining whether traits are the products of selection, but despite our focus on individual traits we emphasize that selection works on whole phenotypes. Nowhere have we suggested that traits or states of traits are fit or not fit; rather, we have argued that traits have fitness values. We have suggested that a design study of phenotypic features and a close tracking of the life history of the feature can yield significant results concerning adaptation, but at some point we can move beyond asking functional, "how-does-it-work" questions and focus on broader issues, placing our knowledge of discrete functions into an organized synthesis of adaptive behavior. 
For example, the changes in ceramic-vessel technology discussed above have been used to track other aspects of human adaptedness in the riverine Midwest during the period 200 B.C.-A.D. 750. Vessel-wall-thickness curves for western Illinois (Braun 1985b, 1987), eastern Missouri (O'Brien and Holland 1991), and southern Illinois (Hargrave 1981) show that around A.D. 50 vessel walls began at least a 700 -year-long decline in mean thickness. Braun $(1985 \mathrm{~b}, 1987)$ infers that adjustments in pottery technology track changes that took place among Woodland groups producing the vessels changes inferred from the archaeological record to be increased sedentariness (Asch 1976; O'Brien 1987), shifts in household composition and size (Braun 1987), population growth (Buikstra et al. 1986; Holland 1989; O’Brien 1987), increased localization of resource procurement (Styles 1981), and increased nutritional dependence on the seeds of starchy and oily annuals (Asch and Asch 1985). In coevolutionary fashion, Woodland pottery technology shifted, coincident with increased use of seed plants, toward production of thinner-walled vessels that could be used to extract nutritional value from the seeds. It has been suggested (Buikstra et al. 1986; O'Brien 1987) that such a technology could have led to an increase in fertility, as babies were shifted to starchy gruels at an earlier age, thereby decreasing the birth interval. The findings of Buikstra et al. (1986) show that increased fertility did occur during the Late Woodland period.

Concomitant with increased sedentism, localization of resource procurement, increased use of starchy seeds, and changes in cooking-vessel technology, a dramatic change took place in the treatment of the exteriors of cooking jars. Vessels made before ca. 200 B.C. were undecorated except for cordmarking and/or deep incising over most if not all the vessel exteriors. After that date, and for approximately the next 500 years, exteriors of cooking jars often were incised, stamped, and punctated to produce literally dozens of fancy motifs. Remarkably, the same series of designs was produced at many different locales along the Mississippi River and its major tributaries such as the Illinois and Missouri rivers (Braun 1985a). Braun (1986) and Braun and Plog (1982) view this phenomenon in terms of information exchange-part of a social network that distributed risk and benefits among members, transmitted information about the environment to participants, defined lines of fusion and fission for periodic aggregation and dispersion, and delegated decision-making responsibilities (see O'Brien [1987] for extended summary). Whether Braun and Plog are correct on all counts is immaterial. What is important is that they demonstrate that there could have been a distinct adaptive advantage in participating in such a network. And if one of the signifiers of group membership was decorating the exteriors of cooking jars, then decoration was an adaptive feature.

Second, one might question our emphasis on potential fitness rather than on realized fitness. Aren't we interested more in what happened than we are in what might have happened? Yes, we are, though here we are faced with some of the same problems facing paleontologists. How does one adequately demonstrate that possession of a certain feature made an organism more fit than its conspecifics without the feature? Obviously one way would be to show an inequality in the number of offspring produced by each organism (and their successors) in succeeding generations. Archaeologically this would require massive burial data, the size of which probably is unattainable. There is reason to suspect, however, that eventually, with enough data points, it will be possible to infer realized fitness from potential fitness. Engineering-design studies produce the kinds of information needed to create the data points and head us in the right direction, as shown in the example from the Illinois River valley cited above. There, the implied linkage among increased use of starchy seeds, thinner-walled vessels, and increased fertility has paved the way for more-intensive examination of the archaeological record relative to increased realized fitness.

Third, one might ask what the difference is between the kind of approach we advocate and the approach that has a venerated place in anthropology, namely functionalism. This is an interesting question and one that was posed by one of the reviewers. If we accept the term functionalism at face value- understanding how things work - then our approach to adaptation can be termed functional. A selectionist approach, to be anything other than a metaphysical exercise, has to be functional. Darwin, despite claims to the contrary, was a functionalist, i.e., he was fascinated in how things work. Adaptation, if it is anything, is a record of how well organisms function in particular locales at particular times.

So what are the differences between a Darwinian view of function and what has commonly come 
to be known in anthropology as functionalism? Functionalists from Malinowski on down have been interested in how cultures operate, and in several significant respects their ideas and approaches live on in the cultural evolutionists/ecologists of the mid- and late-twentieth century who have taken functional analysis to even greater heights. The close ties between the earlier functionalists and the later cultural evolutionists are clear: "The concept of 'stages' remains as valid as that of origins. We would, however, have to make any evolutionary scheme of successive developmental stages either very general or else valid only for certain regions and under certain conditions" (Malinowski 1960:16). Here Malinowski sounds very much like Steward (e.g., 1955). And at the center of functional analyses has been adaptation. But the ways in which the functionalists/cultural ecologists view adaptation have little in common with the Darwinian view of adaptation. And, as we pointed out earlier, it is the non-Darwinian functionalist view that has pervaded anthropology.

Adaptation, to the functionalists/evolutionists, is a preordained conclusion: "no invention, no revolution, no social or intellectual change, ever occurs except when needs are created and thus new devices or techniques, in knowledge, or in belief are fitted into the cultural processes of an institution" (Malinowski 1960:16). This is about as non-Darwinian a statement as one could make. As Rindos (1989:34) points out, "Functionalists extend to culture the dominant mode of evolutionary explanation used by the nineteenth-century biologist - the very model of environmentally induced variation that Darwin utilized before developing the mature theory in the Origin." As we discussed earlier, Darwin's immature view held that organisms constantly monitor their environments, adjusting only when the environment instructs them to do so, and then only in precisely described manners.

Nowhere is the functionalist approach more apparent in anthropology than in efforts to understand the origins of agriculture-efforts in large part directed toward understanding how and why agriculture started when and where it did. Or, conversely, efforts are expended to understand why it did not begin in a certain area, since plant domestication would seem to have been the "logical" adaptation. Pryor (1986), for example, concludes a study of the origins of agriculture by asking why all societies have not adopted agriculture, since he assumes that over the past 10,000 years four conditions have prevailed: (1) groups had the requisite knowledge for practicing agriculture; (2) hunting and gathering presented such risks that groups should have wanted to adopt some agriculture; (3) in many groups cultural and natural conditions that would have precluded agriculture were small; and (4) population growth would have led to diminishing returns without agriculture. In other words, in the face of risk to a group, it should have, following the functionalist approach, turned to agriculture as the logical adaptation. Researchers such as Pryor are clearly cut from the Steward mold of cultural ecology: "The most fruitful course of investigation would seem to be the search for laws which formulate the interrelationships of particular phenomena which may recur crossculturally but are not necessarily universal" (Steward 1955:29).

A Darwinian approach to anthropology in no way precludes cross-cultural comparisons, though it does preclude, we would argue, searching for "laws which formulate the interrelationships of particular phenomena." The only laws that apply under a Darwinian perspective are laws of contingency. This is not to say that there are not design constraints placed on behaviors as there are on somatic features. It makes little sense, for example, to wonder why igloos were not in use on the Great Plains. But design constraints are not the same as laws that govern outcomes. Searching for functional laws that govern behavior is based on the belief that some nearly perfect ideal exists toward which cultures are pushed. We agree with Rindos (1989:37) that "In calling upon the perfect adaptation of the pre-Origin Darwin, [functionalism] cannot help but posit a cultural adaptationism that is motivated by the generation of directed variation to account for all cultural response to stress." The orthogenetic view of history that guides such attempts is distinctly different from the view we advocate here. Under the functionalist view, if the tape of life is rewound and played a hundred times, the outcomes should be, within reason, almost similar. In other words, human groups would show similar adjustments relative to their adaptive behaviors. Under the Darwinian view, as Gould has pointed out ad nauseum (e.g., Gould 1989), the replays should show a spectacular range of outcomes, with each adaptation contingent in part upon what came before (design constraint) but open to countless possibilities. 


\section{CONCLUSIONS}

Taylor (1948:95) noted over 40 years ago that archaeologists were becoming "as Tolstoy once said of modern historians, like deaf men answering questions which no one has asked them." We have no doubt that much of what we have said in this paper will be perceived in a similar light. But the questions have been asked, even if we have not always heard them so clearly. And at the center of the questions is the concept of adaptation. As Kirch (1980:101) points out, a long-standing aim of anthropology has been to explain "man's myriad adaptations to the environmental challenges of existence." This goal is both valid and worthy of attention, though in practice the role of adaptation too often has been confined to a post-facto "explanation" of the appearance and persistence of a particular trait. We firmly believe that it is not altogether unreasonable to begin with a more-rigorous definition of what adaptation is and, by extension, what it is not.

In defining adaptation, we take our lead from evolutionary biology. To most biologists, an adaptation not only must contribute to an organism's adaptedness, but it must have a pedigree molded by selection. Thus an innovation or invention (or accident) cannot be an adaptation. It is only after an adaptive innovation is replicated (and thus ceases to be an innovation) and is subsequently shaped by selection that an adaptation exists. Use of this more rigorously defined concept of adaptation helps to unravel the archaeological knot composed of conflating style and function. Within the framework offered by this definition, functional traits are those that affect adaptedness, but only those with a history shaped by selection are considered adaptations. On the other hand, style is regarded as more than merely the absence of function. A stylistic trait is one that neither affects adaptedness nor is under selective control.

The problem facing archaeologists interested in adaptation (and most profess to be) would appear to be twofold. First, it is necessary to identify which archaeological features contributed to the adaptedness of the human group(s) under study. This procedure is linked directly to an analytical separation of potential adaptedness from realized adaptedness. Second, archaeologists are obliged to chart the evolutionary history of a particular feature in order to decide whether it has been shaped by selection, since then and only then can a trait be considered an adaptation. Determining whether or not a trait was (is) adaptive is not always easy. For example, adaptations may be nested in a number of hierarchical units, and it is necessary first to adjust the level of analysis to the proper unit. It then may be possible to determine the potential adaptedness of a trait (or suite of traits). Engineering studies are a viable means of assessing fitness, and we suggest elsewhere (O'Brien and Holland 1991) that perhaps this is the most-useful role that the field of archaeometry can play.

The archaeological record, composed mainly of fossilized evidence of successful variants (and which has been disturbed by numerous cultural and natural forces), cannot be assumed to hold all the evidence needed to understand completely prehistoric adaptedness and adaptation. Yet, despite methodological problems, archaeology is well poised to make significant contributions to the study of adaptation. Indeed, archaeologists have a unique laboratory in which to explore human adaptation over an expanse of time not available to the biologist. We need not be, as Taylor warned, like deaf men. The past speaks in a loud voice, but it is up to us to use solid theory, sound analysis, and logical inference to hear the words. But neither can we, as Lewontin (1986:235) warns, act like vulgarizers who by crossing over "the narrow line that separates a genuinely fruitful and powerful theory from its sterile caricature ... seize upon the powerful explanatory element and, by its indiscriminate use, destroy its usefulness." That powerful element is the concept of adaptation.

Acknowledgments. We thank J. Marks, H. C. Wilson, R. C. Dunnell, R. L. Bettinger, H. Neff, R. Boyd, J. J. Reid, and T. Majewski for numerous helpful insights on the manuscript. The shape of the fall-off curve for traits and states under selection was recognized by W. Durham; Dunnell pointed out differences between randomness and stochastic processes; Wilson proposed the example in Note 3; and Reid suggested the distinction between function and functionalism. Axel E. Nielsen prepared the Spanish abstract.

\section{REFERENCES CITED}

Alland, A., Jr.

1975 Adaptation. Annual Review of Anthropology 4:59-73. 
Asch, D. L.

1976 The Middle Woodland Population of the Lower Illinois Valley: A Study in Paleodemographic Models. Scientific Papers No. 1. Northwestern University Archeological Program. Evanston, Illinois.

Asch, D. L., and N. B. Asch

1985 Prehistoric Plant Cultivation in West-Central Illinois. In The Nature and Status of Ethnobotany, edited by R. I. Ford, pp. 149-203. Anthropological Papers No. 75. Museum of Anthropology, University of Michigan, Ann Arbor.

Bethell, T.

1976 Darwin's Mistake. Harper's February:70-75.

Bock, W. J.

1980 The Definition and Recognition of Biological Adaptation. American Zoologist 20:217-227.

Braidwood, R.

1967 Prehistoric Men. 7th ed. Scott, Foresman, Glenview, Illinois.

Brandon, R. N.

1978 Adaptation and Evolutionary Theory. Studies in History and Philosophy of Science 9:181-206.

1990 Adaptation and Environment. Princeton University Press, Princeton, New Jersey.

Braun, D. P.

1977 Middle Woodland-Early Late Woodland Social Change in the Prehistoric Central Midwestern U.S.

Ph.D. Dissertation, University of Michigan. University Microfilms, Ann Arbor.

1983 Pots as Tools. In Archaeological Hammers and Theories, edited by J. Moore and A. Keene, pp. 107134. Academic Press, New York.

1985a Ceramic Decorative Diversity and Illinois Woodland Regional Integration. In Decoding Prehistoric Ceramics, edited by B. A. Nelson, pp. 128-153. Southern Illinois University Press, Carbondale.

1985b Absolute Seriation: A Time-Series Approach. In For Concordance in Archaeological Analysis: Bridging Data Structure, Quantitative Technique, and Theory, edited by C. Carr, pp. 509-539. Westport, Kansas City, Missouri.

1986 Midwestern Hopewellian Exchange and Supralocal Interaction. In Peer-Polity Interaction and Sociopolitical Change, edited by C. Renfrew and J. Cherry, pp. 117-126. Cambridge University Press, New York.

1987 Coevolution of Sedentism, Pottery Technology, and Horticulture in the Central Midwest, 200 B.C.A.D. 600. In Emergent Horticultural Economies of the Eastern Woodlands, edited by W. F. Keegan, pp. 153-181. Occasional Paper No. 7. Center for Archaeological Investigations, Southern Illinois UniversityCarbondale.

1990 Selection and Evolution in Nonhierarchical Organization. In The Evolution of Political Systems: Sociopolitics in Small-Scale Sedentary Societies, edited by S. Upham, pp. 62-86. Cambridge University Press, New York.

Braun, D. P., and S. Plog

1982 Evolution of "Tribal" Social Networks: Theory and Prehistoric North American Evidence. American Antiquity 47:504-525.

Buikstra, J. E., L. Koningsberg, and J. Bullington

1986 Fertility and the Development of Agriculture in the Prehistoric Midwest. American Antiquity 51:528546.

Burian, R.

1983 Adaptation. In Dimensions of Darwinism, edited by M. Grene, pp. 287-314. Cambridge University Press, New York.

Campbell, B. G.

1966 Human Evolution: An Introduction to Man's Adaptation. Aldine, Chicago.

Darwin, C.

1859 On the Origin of Species by Means of Natural Selection, or the Preservation of Favored Races in the Struggle for Life. Murray, London.

Dobzhansky, T.

1982 Genetics and the Origin of Species. Columbia University Press, New York.

Dobzhansky, T., F. J. Ayala, G. L. Stebbins, and J. W. Valentine

1977 Evolution. Freeman, San Francisco.

Dunnell, R. C.

1971 Systematics in Prehistory. The Free Press, New York.

1978a Style and Function: A Fundamental Dichotomy. American Antiquity 43:192-202.

1978b Archaeological Potential of Anthropological and Scientific Models of Function. In Archaeological Essays in Honor of Irving B. Rouse, edited by R. C. Dunnell and E. S. Hall, Jr., pp. 41-73. Mouton, The Hague.

1980 Evolutionary Theory and Archaeology. In Advances in Archaeological Method and Theory, vol. 3, edited by M. B. Schiffer, pp. 35-99. Academic Press, New York.

1985 Methodological Issues in Contemporary Americanist Archaeology. In Proceedings of the 1984 Biennial Meeting of the Philosophy of Science Association, vol. 2, edited by P. D. Asquith and P. Kitcher, pp. 717744. Philosophy of Science Association, East Lansing, Michigan.

1988 Archaeology and Evolutionary Theory. Ms. on file, Department of Anthropology, University of Missouri-Columbia. 
Dunnell, R. C., and J. K. Feathers

1991 Late Woodland Manifestations of the Malden Plain, Southeast Missouri. In Stability, Transformation, and Variation: The Late Woodland Southeast, edited by M. S. Nassaney and C. R. Cobb, pp. 21-45. Plenum, New York.

Feathers, J. K.

1988 Explaining the Transition from Sand to Shell Temper in Southeastern Missouri Pottery. Ms. on file, Department of Anthropology, University of Missouri-Columbia.

1989 Effects of Temper on Strength of Ceramics: Response to Bronitsky and Hamer. American Antiquity 54:579-588.

Fisher, D. C.

1985 Evolutionary Morphology: Beyond the Analogous, the Anecdotal, and the Ad Hoc. Paleobiology 11: $120-138$.

Futuyma, D. J.

1979 Evolutionary Biology. 1st ed. Sinauer, Sunderland, Massachusetts.

1986 Evolutionary Biology. 2nd ed. Sinauer, Sunderland, Massachusetts.

Ghiselin, M. T.

1966 On Semantic Pitfalls of Biological Adaptation. Philosophy of Science 33:147-153.

1969 The Triumph of the Darwinian Method. University of California Press, Berkeley, California.

Gould, S. J.

1976 Darwin's Untimely Burial. Natural History 85:24-30.

1989 Wonderful Life: The Burgess Shale and the Nature of History. Norton, New York.

Gould, S. J., and R. Lewontin

1979 The Spandrels of San Marco and the Panglossian Paradigm: A Critique of the Adaptationist Programme. Proceedings of the Royal Society of London B205:581-598.

Gould, S. J., and E. Vrba

1982 Exaptation-A Missing Term in the Science of Form. Paleobiology 8:4-15.

Gould, S. J., D. M. Raup, J. J. Sepkoski, Jr., T. J. M. Schopf, and D. S. Simberloff

1977 The Shape of Evolution: A Comparison of Real and Random Clades. Paleobiology 3:23-40.

Grimshaw, R.

1971 The Chemistry and Physics of Clays. Ernest Benn, London.

Hargrave, M. L.

1981 Woodland Ceramic Chronometry and Occupational Intensity at the Carrier Mills Archaeological District, Saline County, Illinois. Unpublished Master's thesis, Department of Anthropology, Southern Illinois University-Carbondale.

Holland, T. D.

1989 Fertility in the Prehistoric Midwest: A Critique of Unifactorial Models. American Antiquity 54:389426.

Kirch, P. V.

1980 The Archaeological Study of Adaptation: Theoretical and Methodological Issues. In Advances in Archaeological Method and Theory, vol. 3, edited by M. B. Schiffer, pp. 101-156. Academic Press, New York.

Krimbas, C. B.

1984 On Adaptation, Neo-Darwinian Tautology, and Population Fitness. Evolutionary Biology 17:1-57.

Leonard, R. D., and G. T. Jones

1987 Elements of an Inclusive Evolutionary Model for Archaeology. Journal of Anthropological Archaeology 6:199-219.

Lewontin, R. C.

1978 Adaptation. Scientific American 239:156-169.

1986 Adaptation. Reprinted in Conceptual Issues in Evolutionary Biology: An Anthology, edited by E. Sober, pp. 235-251. MIT Press, Cambridge.

Malinowski, B.

1960 A Scientific Theory of Culture. Oxford University Press, New York.

Malthus, T. R.

1798 An Essay on the Principle of Population. Johnson, London.

Marks, J., and E. Staski

1988 Individuals and the Evolution of Biological and Cultural Systems. Human Evolution 3:147-161.

Mayr, E.

1973 Populations, Species, and Evolution. Harvard University Press, Cambridge.

1982 The Growth of Biological Thought. Harvard University Press, Cambridge.

1988 Toward a New Philosophy of Biology: Observations of an Evolutionist. Harvard University Press, Cambridge.

Million, M. G.

1975 Ceramic Technology of the Nodena Phase People (ca. AD 1400-1700). Southeast Archaeological Conference Bulletin 18:201-208.

Mills, S., and J. Beatty

1979 The Propensity Interpretation of Fitness. Philosophy of Science 46:263-286. 
Neff, $\mathrm{H}$.

1992 Ceramics and Evolution. In Archaeological Method and Theory, vol. 4, edited by M. B. Schiffer. University of Arizona Press, Tucson, in press.

O’Brien, M. J.

1987 Sedentism, Population Growth, and Resource Selection in the Woodland Midwest: A Review of Coevolutionary Developments. Current Anthropology 28:177-197.

O'Brien, M. J., and T. D. Holland

1990 Variation, Selection, and the Archaeological Record. In Archaeological Method and Theory, vol. 2, edited by M. B. Schiffer, pp. 31-79. University of Arizona Press, Tucson.

1991 Evolutionary Selectionism and the Role of Archaeology. Paper presented at the 56th Annual Meeting of the Society for American Archaeology, New Orleans.

Ospovat, D.

1981 The Development of Darwin's Theory. Cambridge University Press, New York.

Pryor, F. L.

1986 The Adaptation of Agriculture: Some Theoretical and Empirical Evidence. American Anthropologist 88:879-897.

Reid, K.

1984 Fire and Ice: New Evidence for the Production and Preservation of Late Archaic Fiber-Tempered Pottery in the Mid-Latitude Lowlands. American Antiquity 49:55-76.

Reynolds, V.

1984 The Relationship Between Biological and Cultural Evolution. Journal of Human Evolution 13:71-79.

Rindos, D.

1984 The Origins of Agriculture: An Evolutionary Perspective. Academic Press, New York.

1985 Darwinian Selection, Symbolic Variation, and the Evolution of Culture. Current Anthropology 26:65-88.

1986 The Evolution of the Capacity for Culture: Sociobiology, Structuralism, and Cultural Selection. Current Anthropology 27:315-332.

1989 Undirected Variation and the Darwinian Explanation of Cultural Change. In Archaeological Method and Theory, vol. 1, edited by M. B. Schiffer, pp. 1-45. University of Arizona Press, Tucson.

Ruse, $M$.

1971 Functional Statements in Biology. Philosophy of Science 38:87-95.

Rye, O. S.

1976 Keeping Your Temper Under Control: Materials and the Manufacture of Papuan Pottery. Archaeology and Physical Anthropology in Oceania 11:106-137.

Schiffer, M. B., and J. M. Skibo

1987 Theory and Experiment in the Study of Technological Change. Current Anthropology 28:595-622.

Sober, E.

1984 The Nature of Selection: Evolutionary Theory in Philosophical Focus. MIT Press, Cambridge.

Steward, J. H.

1955 Theory of Culture Change. University of Illinois Press, Urbana.

1956 Cultural Evolution. Scientific American 194(5):69-80.

Stimmell, C., R. B. Heimann, and R. G. V. Hancock

1982 Indian Pottery from the Mississippi Valley: Coping with Bad Raw Materials. In Archaeological Ceramics, edited by J. S. Olin and A. D. Franklin, pp. 219-228. Smithsonian Institution Press, Washington, D.C.

Styles, B. W.

1981 Early Late Woodland Subsistence in the Lower Illinois Valley. Scientific Papers No. 8. Northwestern University Archeological Program, Evanston, Illinois.

Taylor, W. W., Jr.

1948 A Study of Archeology. Memoir Series No. 69. American Anthropological Association, Menasha, Wisconsin.

Van Valen, L.

1973 A New Evolutionary Law. Evolutionary Theory 1:1-30.

Williams, M. B.

1986 The Logical Status of the Theory of Natural Selection and Other Evolutionary Consequences. Reprinted in Conceptual Issues in Evolutionary Biology: An Anthology, edited by E. Sober, pp. 83-98. MIT Press, Cambridge.

Wobst, H. M.

1977 Stylistic Behavior and Information Exchange. In For the Director: Research Essays in Honor of James B. Griffin, edited by C. E. Cleland, pp. 317-342. Anthropological Papers No. 61. Museum of Anthropology, University of Michigan, Ann Arbor. 


\section{NOTES}

1 "Struggle for existence" is perhaps not the best of terms, but Darwin (1859:62) noted he was using it in a "large and metaphorical sense."

2 Sober (1984) makes a strong argument for the force concept, which is acceptable as long as one keeps in mind that the "force" rarely is constant. But in so doing one also has to refer to mutation and drift as evolutionary forces.

${ }^{3}$ We recognize another possibility. Suppose, for the sake of argument, that wearing skins diminishes the amount of sunlight needed to synthesize vitamin D and the population dies out. Thus, over time, the "adaptation" becomes maladaptive. This exemplifies organismal response lagging behind the source of selection.

${ }^{4}$ We are not suggesting a cooking pot has a phenotype; in reality it the user of the pot who is polymorphic. Nor are we suggesting that pots really have fitnesses except in terms of replicative success. The important point is that replicative success of pots may affect human adaptedness.

Received March 19, 1991; accepted August 22, 1991 